\title{
Throughput Enhancement in OFDM System using Low Overhead Pilot Insertion Scheme over Slow Fading Rayleigh Channel
}

\author{
Atif Sardar Khan \\ University of Engineering \& \\ Technology, Peshawar- \\ Pakistan
}

\author{
Syed Waqar Shah \\ University of Engineering \& \\ Technology, Peshawar- \\ Pakistan
}

\author{
Latif Ullah Khan \\ University of Engineering \& \\ Technology, Peshawar- \\ Pakistan
}

\begin{abstract}
Orthogonal Frequency Division Multiplexing (OFDM) is a widespread adopted technique for wireless and wired communication systems because of its inherent feature of the spectral efficiency and robustness to channel impairments. The throughput deterioration of the OFDM system due to pilots can be reduced by minimizing the density of pilots. A Modified Minimum Mean Square Error (M-MMSE) estimator with low computational complexity based on Low overhead Pilot Insertion (LPI) scheme is also proposed. The pilot overhead in the LPI scheme is reduced compared to blocktype channel estimation. The comparative analysis of the MMMSE estimator for the LPI scheme with MMSE estimator for block-type pilot arrangement shows significant performance improvement of the proposed channel estimation algorithm.
\end{abstract}

\section{Keywords}

OFDM, MMSE Estimator, Block-type Channel estimation, ICI, Equalization

\section{INTRODUCTION}

Widespread applications of the Orthogonal Frequency Division Multiplexing (OFDM) are because of its spectral effectiveness and robustness to frequency selective fading channel impairments. 802.11 based Wireless LANs, Long Term Evolution (LTE) and power line communications use OFDM [1]-[3]. In OFDM, the entire channel bandwidth is divided into sub channels with the objective to transform the frequency selective fading channel into flat fading channel. In the frequency domain due to overlapping of orthogonal subcarriers, the OFDM exhibit spectral efficiency. For the cancellation of Inter Symbol Interference (ISI), complex time domain equalization techniques were adopted while dealing with single carrier communication system. On the other hand, for ISI mitigation in OFDM cyclic prefix is used [4].

In OFDM, the channel estimation and equalization are adopted to mitigate the deterioration caused by the frequency selective fading channel in the presence of Additive White Gaussian Noise (AWGN). There are two types of one dimensional (1-D) channel estimation depending on the placement of pilots per OFDM symbol [5]. Block-type and comb-type channel estimation are used for slow fading and fast fading channels respectively where the channel impulse response has rapid variations. Sensitivity of the OFDM to time and frequency offset errors are its major downsides. Frequency offset errors disturb the alignment of the orthogonal subcarriers which results in Inter Carrier
Interference (ICI). In [6], an interpolation technique has been proposed for comb-type channel estimation over fast fading Rayleigh channel. Second order and time domain interpolation technique were compared with the interpolation technique of [7]. The later outperforms time domain and second order interpolation. In [8], a novel interpolation technique has been proposed for comb-type channel estimation using LS estimator for OFDM system over fast fading Rayleigh channel. The adopted technique of interpolation gives better output than the low pass, spline and linear interpolation. The Least Square (LS) estimator has low computational complexity and does not require a power delay profile for its operation; however, its performance is degraded than MMSE estimator [9]. In [10], a robust modified MMSE (M-MMSE) estimator is proposed for fast fading Rayleigh channel. Comparative analysis of M-MMSE and MMSE estimator with linear, low pass and spline interpolation reveals the performance improvement of M-MMSE estimator over MMSE estimator. Throughput deterioration is caused by pilots used for channel estimation in OFDM systems. Therefore, measures should be taken in order to reduce the pilot overhead for improving the throughput performance of the OFDM system.

In this paper, modifications in the MMSE estimator for the LPI scheme are proposed. The modification results in reduction of the computational complexity by reducing the size of the channel auto-covariance and cross-covariance matrices respectively. The pilot overhead is reduced in LPI scheme compared to block-type channel estimation. Comparative analysis of the proposed M-MMSE estimator for the LPI scheme with MMSE estimator for block-type channel estimation through simulation results show the performance improvement of the proposed channel estimation algorithm.

The remaining content of the paper goes as: Section II introduces the OFDM system model with mathematical manipulations. Section III discusses MMSE estimator for block-type channel estimation. Section IV explains the LPI scheme and modified MMSE estimator. simulation result analyses has been carried out in section whereas, Section VI concludes the final paper.

Notations: Bold and uppercase italic style represents matrices; Lower case italic style represents vectors. Hertmitian transpose is represented by superscript ' $H$ ' and transpose is represented by superscript ' $\mathrm{T}$ '. * denotes the complex conjugate. $\mathrm{E}\{\}$ denotes the expectation. $\operatorname{Diag}(\boldsymbol{x})$ represents the elements of $\boldsymbol{x}$ on its diagonal. 


\section{OFDM SYSTEM MODEL}

First of all, the input bit sequence in the OFDM system is generated as shown in Fig. 1. The resulting sequence is mapped and then changed into sizeable groups equal to the subcarriers $N_{d}$ reserved for data per OFDM symbol and, then parallelized. The $i^{\text {th }}$ group of symbols is given by:

$$
\boldsymbol{s}^{i}=\left[s^{i}(0), s^{i}(1), \ldots, s^{i}\left(N_{d}-1\right)\right]^{T}
$$

After parallelization, pilot symbols are inserted into OFDM symbols according to the comb-type channel estimation and LPI scheme respectively. The pilot added $i^{\text {th }}$ group of symbol $\boldsymbol{d}^{i}=\left[d^{i}(0), d^{i}(1), \ldots, d^{i}(N-1)\right]^{T}$ is then converted into time domain by the operation of the Inverse Discrete Fourier Transform (IDFT).

$$
c^{i}=F^{H} d^{i}
$$

Where $[\boldsymbol{F}] n, n=e^{\frac{-j 2 \pi(n-1)(n-1)}{N}}$ for $n=0,1,2, \ldots, N-1$. Guard interval of type cyclic prefix is added to the OFDM symbol in order to cancel out ISI. The length of the cyclic prefix should be kept greater than the highest delay spread of the multipath fading channel for effective mitigation of ISI. The cyclic prefix added $i^{\text {th }}$ OFDM symbol is given by:

$$
\begin{aligned}
\boldsymbol{c}_{C P}{ }^{(i)}= & {\left[c^{(i)}\left(N-L_{C P}\right), c^{(i)}\left(N-L_{C P}+1\right), \ldots, c^{(i)}(N-\right.} \\
& \left.\left.2 L_{C P}+1\right), c^{(i)}(0), c^{(i)}(1), \ldots, c^{(i)}(N-1)\right]^{T}(3)
\end{aligned}
$$

Where $L_{C P}$ is the length of the cyclic prefix. Finally, the signal in the presence of AWGN are passed through a multipath fading channel. The signal at the receiver side after removal of the cyclic prefix is transformed into the frequency domain by the operation of the DFT.

$$
\boldsymbol{r}^{i}=\boldsymbol{F} \tilde{\boldsymbol{c}}^{i}
$$

Equalization is performed after channel estimation. Finally, the signal is passed through the demapper to yield the output bits.

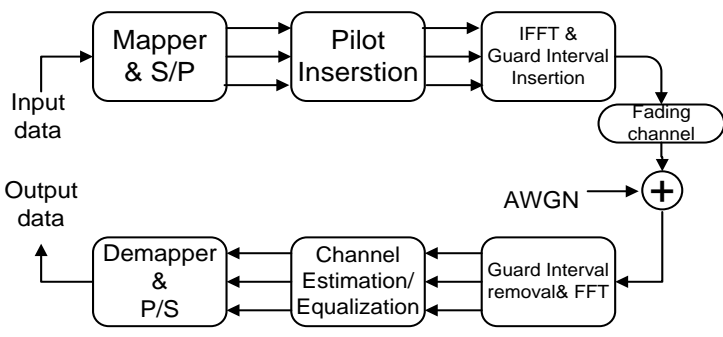

Fig 1: OFDM System Model

\section{MMSE ESTIMATOR BASED ON BLOCK-TYPE PILOT ARRANGEMENT}

Block-type channel estimation is used for slow fading channels where variations in the channel impulse response are slow. The arrangement of pilot tones in block-type channel estimation is illustrated in Figure 2. The channel impulse response is assumed constant for a block period of time for block-type channel estimation. The resulting estimated frequency response of the channel for the first symbol is then adopted as a default estimated channel frequency response for the proceeding symbols in a block. The reason for transmitting the pilot tones in all subcarriers of the first symbol is to avoid the interpolation error inherent in every interpolation technique. The channel frequency response at pilot tones is estimated through the use of MMSE estimator because of its optimum performance with a sense of Mean
Square Error (MSE) [5]. In frequency domain, the MMSE estimate of the channel for a channel vector $h$ in the time domain of Gaussian distribution and having no correlation with channel noise is given by [11]:

$$
\boldsymbol{h}_{M M S E}=\boldsymbol{F}_{N}{ }^{H} \boldsymbol{R}_{H Y} \boldsymbol{R}_{Y Y}{ }^{-1} \boldsymbol{r}_{P}
$$

Where

$\boldsymbol{F}_{N}$ is the $N X N$ matrix $\left[\boldsymbol{F}_{N}\right] n, n=\frac{1}{\sqrt{N}} e^{j 2 \pi(n-1)(n-1) / N}$

$\boldsymbol{R}_{Y Y}=E\left\{\boldsymbol{r}_{P} \boldsymbol{r}_{P}{ }^{H}\right\}=\operatorname{Diag}\left(\boldsymbol{x}_{P}\right) \boldsymbol{F}_{N}{ }^{H} \boldsymbol{R}_{H H} \boldsymbol{F}_{N} \operatorname{Diag}\left(\boldsymbol{x}_{P}\right)^{H}+$ $\sigma^{2} \boldsymbol{I}_{N}$ is the auto-covariance matrix of the $\boldsymbol{r}_{P}$.

$\boldsymbol{R}_{H Y}=E\left\{\boldsymbol{h}_{P} \boldsymbol{r}_{P}{ }^{H}\right\}=\boldsymbol{R}_{H H} \boldsymbol{F}_{N} \operatorname{Diag}\left(\boldsymbol{x}_{P}\right)^{H}$ is the cross covariance matrix of the channel vector $\boldsymbol{h}$ in time domain and $\boldsymbol{r}_{P}$.

$\sigma^{2}$ is the noise variance and $\boldsymbol{R}_{H H}$ is the auto-covariance matrix of $\boldsymbol{h}$.Rewrite (5) as:

$$
\boldsymbol{h}_{M M S E}=\boldsymbol{F}_{N}{ }^{H} \boldsymbol{G}_{M M S E} \boldsymbol{F}_{N} \operatorname{Diag}\left(\boldsymbol{x}_{P}\right)^{H} \boldsymbol{r}_{P}
$$

Where

$$
\begin{aligned}
& \boldsymbol{G}_{M M S E} \\
& =\boldsymbol{R}_{H H}\left[\left(\boldsymbol{F}_{N} \operatorname{diag}\left(x_{P}\right)^{H} \operatorname{Diag}\left(\boldsymbol{x}_{P}\right) \boldsymbol{F}_{N}{ }^{H}\right)^{-1} \sigma^{2}\right. \\
& \left.+\boldsymbol{R}_{H H}\right]^{-1}\left(\boldsymbol{F}_{N} \operatorname{diag}\left(\boldsymbol{x}_{P}\right)^{H} \operatorname{Diag}\left(\boldsymbol{x}_{P}\right) \boldsymbol{F}_{N}{ }^{H}\right)^{-1}
\end{aligned}
$$

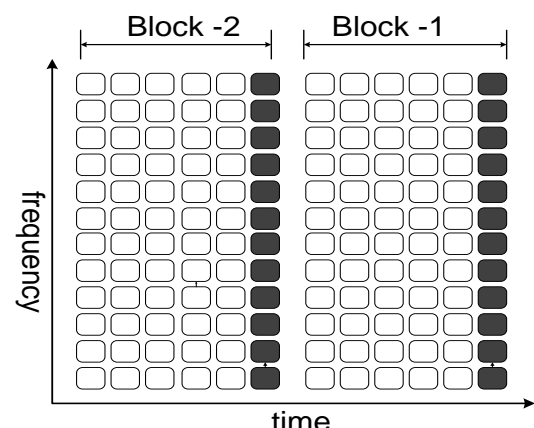

Pilot Subcarrier

Data Subcarrier

Fig 2: Pilot Arrangement in Block-type Channel Estimation

\section{MODIFIED MMSE ESTIMATOR BASED ON PROPOSED PILOT ARRANGEMENT}

Design of an estimator with low computational complexity and low pilot overhead is a challenging task. In this paper, a novel pilot insertion scheme for OFDM system over the slow fading channel is proposed. In the time domain, the energy of the channel impulse response is concentrated [12]. No energy leakage was observed in case of sample spaced channel and all the fading impulses are located at an integer multiples of the system sampling rate. The energy of the channel impulse response is concentrated in the first $L \leq L_{C P}$, where $L$ is the channel order and $L_{C P}$ is the cyclic prefix length as the channel impulse response is contained within the first $L$ taps less than or equal to the guard interval. Therefore, the channel impulse response can be written as:

$$
h(n)=\left\{\begin{array}{cl}
\operatorname{IFFT}(H(K)), & 0 \leq n \leq L-1 \\
0 & L \leq n \leq N-1
\end{array}\right.
$$


Where $H(K)$ is the channel frequency response at $\mathrm{k}^{\text {th }}$ subcarrier. The channel energy is concentrated in time domain and distributed in frequency domain. Therefore, pilot overhead can be reduced by transmitting the pilot symbols only on the P taps equal to or greater than the channel order. The proposed pilot insertion scheme for slow fading channels is shown in Fig 3. The pilot symbols are solely inserted at dedicated subcarriers into the first OFDM symbol and then, The resulting output frequency of the channel for the first symbol is then adopted as a default estimated channel frequency response for the proceeding symbols equalization in OFDM as shown in Fig. 3.

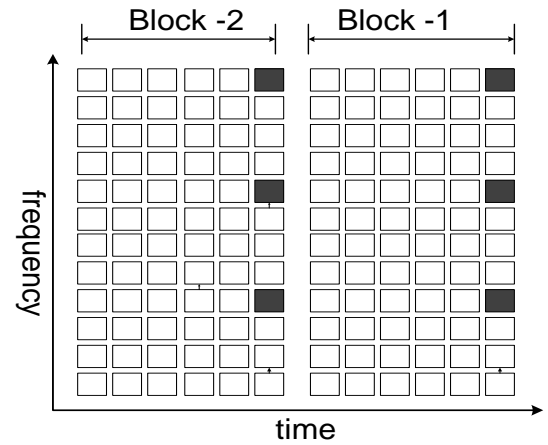

Pilot Subcarrier

Data Subcarrier

Fig 3: LPI Scheme

The M-MMSE estimate of the channel in frequency domain for a time domain channel vectorh of Gaussian distribution and uncorrelated with channel noise is given by:

$$
\boldsymbol{h}_{M-M M S E}=\boldsymbol{F}_{M-N}{ }^{H} \boldsymbol{R}_{M-H Y} \boldsymbol{R}_{M-Y Y}{ }^{-1} \boldsymbol{r}_{M-P}
$$

Where

$\boldsymbol{F}_{M-N}$ is the $L X N$ matrix $\left[F_{M-N}\right] l, n=e^{j 2 \pi(l-1)(n-1) / N}$

Where $L$ is the channel order.

$\boldsymbol{r}_{M-P}$ is the vector containing the received pilots at dedicated subcarriers for the proposed LPI scheme.

$\boldsymbol{x}_{M-P}$ is the vector containing the transmitted pilots at dedicated subcarriers for the proposed LPI scheme.

$\boldsymbol{R}_{M-Y Y}=E\left\{\boldsymbol{r}_{M-P} \boldsymbol{r}_{M-P}{ }^{H}\right\}=$

$\operatorname{Diag}\left(\boldsymbol{x}_{M-P}\right) \boldsymbol{F}_{M-N}^{H} \boldsymbol{R}_{M-H H} \boldsymbol{F}_{M-N} \operatorname{Diag}\left(\boldsymbol{x}_{M-P}\right)^{H}+\sigma^{2} \boldsymbol{I}_{L}$ is the auto-covariance matrix of the received signal vector $\boldsymbol{r}_{M-P}$ containing pilots for the proposed LPI scheme.

$\boldsymbol{R}_{M-H Y}=E\left\{\boldsymbol{h}_{M-P} \boldsymbol{r}_{M-P}{ }^{H}\right\}=\boldsymbol{R}_{M-H H} \boldsymbol{F}_{M-N} \operatorname{Diag}\left(\boldsymbol{x}_{M-P}\right)^{H}$ is the cross covariance matrix of the channel vectorh in time domain and $\boldsymbol{r}_{M-P}$.

$\sigma^{2}$ is the noise variance and $\boldsymbol{R}_{M-H H}$ is the auto-covariance matrix of $\boldsymbol{h}$. Rewrite (8) as:

$\boldsymbol{h}_{M-M M S E}=\boldsymbol{F}_{M-N}{ }^{H} \boldsymbol{G}_{M-M M S E} \boldsymbol{F}_{M-N} \operatorname{Diag}\left(\boldsymbol{x}_{M-P}\right)^{H} \boldsymbol{r}_{M-P}(9)$

Where

$\boldsymbol{G}_{M-M M S E}$

$=\boldsymbol{R}_{M-H H}\left[\left(\boldsymbol{F}_{M-N} \operatorname{Diag}\left(x_{M-P}\right)^{H} \operatorname{Diag}\left(\boldsymbol{x}_{M-P}\right) \boldsymbol{F}_{M-N}{ }^{H}\right)^{-1} \sigma^{2}\right.$

$\left.+\boldsymbol{R}_{M-H H}\right]^{-1}\left(\boldsymbol{F}_{M-N} \operatorname{Diag}\left(\boldsymbol{x}_{M-P}\right)^{H} \operatorname{Diag}\left(\boldsymbol{x}_{M-P}\right) \boldsymbol{F}_{M-N}{ }^{H}\right)^{-1}$

\section{SIMULATION RESULTS \& ANALYSIS}

In this section, performance of the proposed channel estimation algorithm and MMSE estimator for block-type pilot insertion has been evaluated using MATLAB. Table I lists the Simulation parameters. The channel adopted in the current scenario is characterized by L complex taps having zero mean and Gaussian distribution.

Table 1. Simulation Parameters

\begin{tabular}{ll}
\hline \hline Parameter & Values \\
\hline Total Subcarrier & 512 \\
Channel Estimation & MMSE and M-MMSE \\
Channel & Slow Fading Rayleigh \\
Modulation schemes & BPSK \& QPSK \\
\hline \hline
\end{tabular}

The simulated frame structure is shown in Fig. 4. In this case, seven symbols constitute the frame unit having first symbol containing pilots entirely for block-type channel estimation, both pilot and data for LPI scheme respectively. The Fig. 5 shows the performance of M-MMSE estimator for the proposed LPI scheme is better than MMSE estimator for block-type pilot arrangement. The complexity comparison of the estimators is also shown in table 2 . Table 2 clearly reveals the reduced complexity of the proposed M-MMSE estimator compared to MMSE estimator. The pilot overhead associated with the proposed LPI scheme is also less than the block-type channel estimation.

Table 2. Complexity Comparison of the Estimators

\begin{tabular}{lll}
\hline \hline Estimator & MMSE & M-MMSE \\
\hline Size of $\mathbf{Q}_{\text {MMSE }}$ & $N X N$ & N.A \\
Size of $\mathbf{G}_{\mathrm{M}-M M S E}$ & N.A & $L X L$ \\
\hline \hline
\end{tabular}

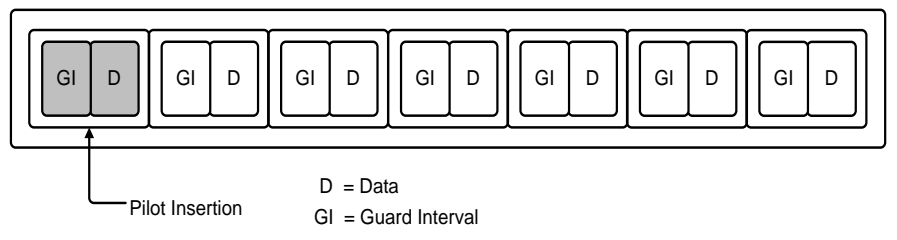

Fig 4: Simulated Frame structure [13] 


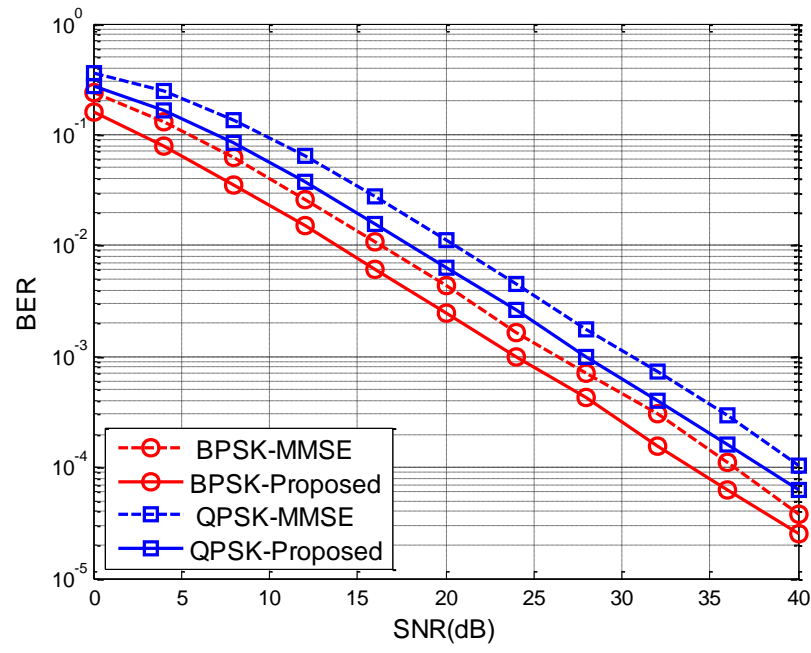

Fig 5: BER Performance curves of MMSE and M-MMSE estimator for different modulation schemes.

\section{CONCLUSIONS}

Modified MMSE estimator based on the LPI scheme is proposed. M-MMSE estimator with LPI scheme outperformed the MMSE estimator with block-type pilot arrangement. There is a reduction in the pilot overhead associated with the proposed LPI scheme compared to block-type pilot arrangement. The computational complexity associated with M-MMSE estimator is also significantly reduced.

\section{REFERENCES}

[1] HuiLiu \& Guoqing Li. 2005. OFDM-based Broadband Wireless Networks, Design and optimization. New jersey: John Wiley \& sons

[2] J. Armstrong, 2012. Tutorial on Optical OFDM, proceedings of the 14th International Conference on Transparent Optical Networks (ICTON)

[3] S. Zeeshan, M. Arif Wahla, M. Babar-Inayatullah, 2011. OFDM, Turbo Codes and Improved Channel Estimation-A Magical Combination, VDM Verilog Publishers, Germany.

[4] Z. Yang, W. Bai, and Z. Liu, 2006. A Decision-Aided Residual ISI Cancellation Algorithm for OFDM Systems, proceedings of the 8th IEEE International Conference on Signal Processing, 16-20.
[5] Hala M.Mahmoud, Allam S.Mousa and Rashid Saleem, 2010. "Channel Estimation Based in Comb-Type Pilots Arrangement for OFDM System over Time Varying Channel," Journal of networks, Vol. 5(7),(july 2010), pp.766-772,

[6] C. He, Z. Peng, Q. Zeng and Y. Zeng, 2009. A Novel OFDM Interpolation Algorithm Based on Comb-Type Pilot, proceedings of the 5th International Conference on Wireless Communications, Networking and Mobile Computing,(Sept. 2009), 1-4.

[7] H. Chunlong, H. li. 2009. Pilot-Aided Channel Estimation Techniques in OFDM System, Proceedings of the International Conference on Communication Software and Networks, (February 2009), 143-146.

[8] L. U. Khan,M. H. Zafar, M. I. Khattak, N. Khan. 2014. A novel channel estimation error minimizing interpolation technique for OFDM systems, proceedings of the 2014 9th International Symposium on Communication Systems, Networks \& Digital Signal Processing (CSNDSP), (July 2014), 716-720.

[9] M. Morelli, U. Mengali, 2001. "A comparison of pilotaided channel estimation methods for OFDM systems," IEEE Transactions on Signal Processing, vol. 49(12), 3065-307.

[10] L. U. Khan, M. Inayatullah Babar, Z. Sabir, 2013. Robust modified MMSE estimator for comb-type channel estimation in OFDM systems, proceedings of the 15th International Conference on Advanced Communication Technology (ICACT), (2013), 924-928.

[11] A.R. James, R.S. Benjamin, S. John, T.M. Joseph, V Mathai, S.S. Pillai, 2011. Channel estimation for OFDM systems. Proceedings of the International Conference on Signal Processing, Communication, Computing and Networking Technologies (ICSCCN), (2011).587-591.

[12] M.-H. Hsieh, and C.-H. Wei, 1998. "Channel Estimation for OFDM Systems based on Comb-type Pilot Arrangement in Frequency Selective Fading Channels. IEEE Transactions on Consumer Electronics, 44(1), (1998), 217-225.

[13] R. Prasad, 2004. OFDM for Wireless Communication Systems, Artech House Inc., Boston, USA. 